\title{
Von der Wissenschaftstheorie zur Soziologie der Wissenschaft
}

\author{
JÖRG NIEWÖHNER
}

Das Forschungsfeld der Science and Technology Studies hat wesentliche Impulse für seine Entstehung und Entwicklung aus der Wissenschaftstheorie, vor allem der ersten Hälfte des 20. Jahrhunderts bezogen. Der logische Positivismus des Wiener Kreises, Karl Poppers kritischer Rationalismus und der Historizismus, entscheidend geprägt von Thomas Kuhn, stellen bis heute die zentralen philosophischen Grundpfeiler der Analyse von Erkenntnisprozessen dar. Alle drei Ansätze untersuchen Erkenntnis mehr oder weniger idealistisch, $d$. h. auf der Ebene von Konzepten, die losgelöst von den sozialen und materiellen Bedingungen ihrer Produktion verstanden werden. Die Untersuchung dieser Produktionsbedingungen war lange Zeit der Wissenschaftsgeschichte vorbehalten. Eine für die Science and Technology Studies prägende Ausnahme in der ersten Hälfte des 20. Jahrhunderts bildet Ludwik Flecks Analyse von Erkenntnis als abhängig von historisch und sozial geformten Denkstilen. Erkenntnisprozesse werden durch Fleck weder idealistisch noch psychologisch gefasst, sondern als fortlaufender Prozess kollektiven sozialen Handelns. Es ist diese Perspektive auf Wissenschaft und Technologieentwicklung, die deutlich macht, wie Wissensproduktion und Erkenntnis zentrale Themen sozialanthropologischer Studien sein können: als situierte, sozial und historisch kontingente Praxis wissenschaftlichen Alltags. 


\section{Weiterführende Literatur}

Young, Allan (1995): The Harmony of Illusions: Inventing Post-Traumatic Stress Disorder, Princeton: Princeton University Press.

Eine anthropologische Untersuchung der Entstehung der Diagnose PostTraumatische Belastungsstörung in den USA in der Folge des Vietnamkriegs. Allan Young greift auf das Fleck'sche Konzept der Harmonie der Täuschung zurück und analysiert umfassend die Entwicklung medizinisch-psychiatrischen Wissens in einem spezifischen Kontext.

Hacking, Ian (1992): »)Style for Historians and Philosophers«. Studies in History and Philosophy of Science Part A, 23(1), 1-20.

Eine wissenschaftshistorische Analyse, die Philosophie und Geschichte über das Konzept des \Stils« zusammenführen möchte. Präzise geschrieben und mit vielen hilfreichen Bezügen zwischen Fleck und zentralen wissenschaftsphilosophischen und-historischen Positionen. 


\section{Einleitung}

Dieser Beitrag zeichnet die Wurzeln des Forschungsfeldes der Science and Technology Studies in der Wissenschaftstheorie des frühen 20. Jahrhunderts nach und verfolgt diese bis in die Anfänge der Wissenschaftssoziologie in den 1950er Jahren. Das Forschungsfeld der Science and Technology Studies, so wie es sich heute darstellt, integriert eine Vielzahl verschiedener Disziplinen: Soziologie und Anthropologie (Europäische Ethnologie), aber auch Wissenschaftsgeschichte und -philosophie, Politik- und Sprachwissenschaften ebenso wie Psychologie. Wichtige theoretische Anregungen gingen zudem aus von interdisziplinären Feldern wie den gender, postcolonial oder cultural studies. Es ist daher keine einfache Aufgabe, den Wurzeln dieses heterogenen Feldes nachzuspüren. Je nach disziplinärer Zugehörigkeit würden eine jede Autorin und ein jeder Autor wohl unterschiedliche theoretische Aspekte, Methoden und Personen hervorheben. Dieser Beitrag ordnet die wissenschaftstheoretischen Entwicklungen aus der Sicht der heutigen Sozial- und Kulturanthropologie. Er stellt drei Kernpositionen der philosophischen Wissenschaftstheorie des 20. Jahrhunderts den Überlegungen des Mediziners Ludwik Fleck gegenüber und betont damit eine sozial wie historisch situierte Perspektive auf Wissen, Denken und Erkenntnis. Diese Herangehensweise betont die Schnittstellen und Anschlussfähigkeiten von Wissenschaftstheorie und anthropologischen Verständnissen von Wissen als Praxis. Es ist aber auch eine spezifisch disziplinäre Lesart der Entwicklungen, die keinesfalls als umfassender Überblick verstanden werden kann. Stattdessen empfiehlt es sich, dieses Kapitel einerseits durch auf Englisch verfügbare Einführungen in die Wissenschaftsforschung und ihre Grundlagen in der philosophy of science zu ergänzen; andererseits sie durch Lektüre einschlägiger wissenschaftstheoretischer und -historischer Texte zu vertiefen.

Bevor sich dieser Beitrag seinem eigentlichen Kern zuwendet, soll einführend die Frage erörtert werden, warum die Entstehung von Wissen überhaupt ein anthropologisches Thema ist. In der Tat entsteht in den folgenden Abschnitten rasch der Eindruck, dass die Entstehung von wissenschaftlichem Wissen ein universelles Phänomen ist, das wenig mit der Spezifik gelebter Alltage und spezifischen - zeitlich und räumlich lokalisierten - Bedingungen zu tun hat. Wissenschaftstheorie ist zuvorderst ein Feld der Philosophie. Ihr Metier ist das abstrakte Konzept, das losgelöst von seinen 
konkreten lokalen, historischen und epistemischen Produktionsbedingungen erörtert werden kann. Aus sozialanthropologischer Perspektive scheint eine solche un-verortete oder nicht-situierte Arbeit an und mit Konzepten ein wenig fremd. Mehr noch: die Vorstellung, man könne über Erkenntnis, Wahrheit und Wissen sprechen, als seien es Konzepte losgelöst von konkreter Praxis, stellt an sich eine hochgradig spezifische, westliche Sichtweise dar. Der US amerikanische Anthropologe Marshall Sahlins hat in seinem wegweisenden Aufsatz »The Sadness of Sweetness. The native Anthropology of Western Cosmology« (Sahlins 1996) deutlich gemacht, bis zu welchem Grad Vorstellungen von Rationalität und Logik und damit auch von Wissen, Wissensproduktion und durch Wissen ermöglichter technologischer Fortschritt spezifisch westlich moderne Phänomene darstellen. Dass wissenschaftliche Methoden zur objektiven Erkenntnis und zur Produktion von wissenschaftlich gesicherter Wahrheit befähigen, d. h. zu objektivem Wissen über die Natur und darauf basierender Technologieentwicklung, ist eine kontingente $-\mathrm{d}$. h. historisch unter spezifischen Bedingungen entstandene, tradierte und weiterentwickelte - Perspektive. Sie hat ihre Wurzeln in der Antike, ist seit der Aufklärung in Euro-Amerika dominant und wird für gewöhnlich für den wissenschaftlich-technologischen Fortschritt in der industriellen Moderne verantwortlich gemacht. Sie ist aber keineswegs in irgendeinem Sinne notwendig die einzige oder die richtige Perspektive. Für westlich geprägte, anthropologisch Forschende im Feld der Science and Technology Studies ist es daher eine zentrale Aufgabe, die spezifisch westlichen Vorannahmen, die die Entwicklung von Wissenschaft und Technologie in unseren Gesellschaften prägen, auf ihre soziale und historische Kontingenz hin zu hinterfragen und aus den Differenzen zu alternativen Ordnungsvorstellungen Erkenntnisse zu gewinnen. Diese Überlegungen bilden in den folgenden Abschnitten einen Subtext und werden in der abschließenden Diskussion von Ludwik Flecks Ansatz zur Analyse wissenschaftlichen Denkens und Handelns explizit relevant gemacht.

Das Forschungsfeld der Science and Technology Studies entsteht in den 1970er Jahren vor allem aus einer Kritik bestehender Ansätze in der Wissenschaftstheorie und der Wissenschaftssoziologie. Um zu verstehen, warum und wie dieses neue Forschungsfeld wissenschaftliche Wissensproduktion und Technologieentwicklung anders analysiert, als bis dahin meist der Fall, ist es daher von Bedeutung, die ihm vorgehenden Forschungsansätze und -ziele zumindest in ihren Grundzügen nachzuvollziehen. Daher widmet 
sich dieses Kapitel der Wissenschaftsphilosophie und hier speziell der Wissenschaftstheorie (und bis zu einem gewissen Grad der von Philosophen betriebenen Wissenschaftsgeschichte), bevor das folgende Kapitel die Wissenschaftssoziologie vor allem in seiner frühen Prägung durch den amerikanischen Soziologen Robert K. Merton näher untersucht. Der Fokus liegt dabei auf der Frage, wie diese Forschungsansätze die Entstehung von Wissen analysieren. Technologieentwicklung und die Geschichte seiner Beforschung wird vor allem in Kapitel vier dieses Bandes zur sozialen Konstruktion von Technologie dargelegt.

Vereinfacht gesagt, befasst sich die Wissenschaftstheorie abstrakt mit dem Erkenntnisprozess in den Wissenschaften und die Wissenschaftssoziologie mit der Entwicklung der Wissenschaft als Institution. Es mag heute Vielen offensichtlich scheinen, dass sich diese beiden Forschungsrichtungen in vielen Fragen nur unzureichend klar voneinander trennen lassen. Denn viele der wegweisenden wissenschaftstheoretischen Debatten des 20. Jahrhunderts sind gerade um dieses Schnittfeld zwischen Epistemologie, Soziologie und Psychologie herum organisiert. Und es ist ein Charakteristikum der Science and Technology Studies, dass sie genau in diesem Schnittfeld zuhause sind, denn sie untersuchen Fragen von Erkenntnis nie abseits von ihren Produktionsbedingungen, sondern sie befragen Produktionsbedingungen von Wissen immer auch in Hinsicht auf ihre epistemologischen Aspekte. Die recht scharfe Trennung von Wissenschaftstheorie und Wissenschaftssoziologie in zwei verschiedene Kapitel ist also überwiegend didaktischen Überlegungen geschuldet. Sie dient der Vereinfachung des ersten Zugriffs und darf keinesfalls als tatsächliche Trennung verstanden werden.

Dieser Beitrag widmet sich den für die Science and Technology Studies (STS) relevanten Grundlagen der Wissenschaftstheorie. Zu diesem Zweck beleuchtet er die Arbeiten von vier Wissenschaftlern genauer, deren Namen weit über die Wissenschaft hinaus geläufig sind: Rudolf Carnap, Karl Popper, Thomas Kuhn und Ludwik Fleck. Carnap, Popper und Kuhn stehen exemplarisch für wichtige Denkrichtungen in der Wissenschaftstheorie des 20. Jahrhunderts. Rudolf Carnap prägte den logischen Positivismus des frühen 20. Jahrhunderts bis hinein in die frühen 1960er Jahre. Er war ein zentraler Vertreter des so genannten »Wiener Kreises« und damit eine Schlüsselfigur der Wiener Moderne. Karl Popper wird gemeinhin dieser Phase des Positivismus zugerechnet, vertrat aber mit seiner Idee der Falsifikation wis- 
senschaftlicher Theorien eine Ausrichtung, die sich in wesentlichen Punkten bewusst vom logischen Positivismus des Wiener Kreises absetzt. Mit Thomas Kuhns berühmt gewordenem Konzept des Paradigmenwechsels begann in den späten 1960er Jahren eine neue Denkrichtung, die als Historizismus in der Wissenschaftsforschung bekannt geworden ist. Ihr gehörten neben Thomas Kuhn auch Paul Feyerabend, Imre Lakatos und Stephen Toulmin an; Namen, die nicht nur in diesem Band, sondern in Veröffentlichungen aus dem Feld der Science and Technology Studies immer wieder auftauchen. Mit Beginn der 1980er Jahre setzten sich in einem dritten Schritt vor allem neue naturalistische und realistische Positionen durch, die bis heute diskutiert werden und die für STS eine wichtige Rolle spielen. Dieser Beitrag beschäftigt sich zunächst ausführlich mit diesen drei Phasen und wendet sich erst dann Ludwik Fleck zu. Fleck veröffentlichte seine Ideen zur Entstehung wissenschaftlicher Tatsachen zwar schon in den 1920er und 30er Jahren, blieb aber bis 1970 weitgehend ungelesen. Die dann einsetzende intensive Auseinandersetzung mit seinem Ansatz machte deutlich, dass Vieles, was Popper, Kuhn und andere entwickelt hatten, bei Fleck bereits ausgearbeitet war - viele Forschende im Feld der Science and Technology Studies würden heute sagen: sogar wesentlich überzeugender ausgearbeitet.

\section{Logischer Positivismus Und Wiener Moderne}

Der logische Positivismus, auch als Neopositivismus bekannt (engl. v.a. logical positivism), stellt eine zentrale wissenschaftstheoretische Position der westlichen Welt des 20. Jahrhunderts dar, die nach dem Ende des ersten Weltkriegs vor allem in Wien und Berlin entwickelt wurde und die eine Weiterentwicklung des Positivismus darstellte, wie er von Auguste Comte und anderen bereits in der ersten Hälfte des 19. Jahrhunderts entworfen worden war. ${ }^{1}$ Zentrale Figur des logischen Positivismus im so genannten

1 Auf den Positivismus allgemein kann in diesem Zusammenhang nicht eingegangen werden. Interessierte, denen diese Denkrichtung bisher nicht vertraut ist, finden in David Hess' »Advanced Introduction to Science Studies« (1997) einen sinnvollen Einstieg. Gleiches gilt für die Abgrenzung des logischen Positivismus von damals verbreiteten naturalistischen Denkrichtungen. 
Wiener Kreis war der Philosoph Rudolf Carnap (*1871 im heutigen Wuppertal - $\dagger 1970$ Santa Monica, Kalifornien). In den Treffen dieses Zirkels von 1922 bis 1936 unter der Leitung von Moritz Schlick entwickelte maßgeblich Carnap auf der Grundlage der Schriften von Bertrand Russell, Alfred North Whitehead und Friedrich Gottlob Frege eine logische Analyse der Wissenschaft(ssprache) (Carnap 1934/1968).

Der logische Positivismus geht davon aus, dass Aussagen nur dann sinnvoll sind, wenn sie verifiziert, d. h. belegt werden können. `Belegen< bedeutet in diesem Kontext, dass Aussagen auf Beobachtungen rückführbar und durch einen rationalen Prozess auf ihren Wahrheitsgehalt hin überprüfbar sind. Anders formuliert: Empirismus und Rationalismus bilden die Grundlage für Unterscheidungen zwischen wahren und falschen Aussagen und damit die Grundlage für Erkenntnis und folglich Wissenschaft. Damit grenzen sich die logischen Positivisten vor allem von theologischen und metaphysischen Begründungen von Erkenntnis ab. Erkenntnis wird untrennbar mit Erkennen im Sinne von Beobachten verbunden. Erkennbar ist nur, was beobachtbar ist. Andere Formen der Erkenntnis, z. B. spiritueller, religiöser oder metaphysischer Art, werden damit als der Wissenschaft nicht zugehörig ausgeschlossen. Diese Trennung wird dadurch zementiert, dass die Herstellung eines Zusammenhangs zwischen Beobachtungen und allgemeinen Aussagen oder Theorien nur auf dem Wege des rationalen Folgerns möglich ist. Rationale und nachvollziehbare Prozedere allein garantieren die gültige Unterscheidung zwischen wahr und falsch, und nicht wie auch immer geartete Glaubenssätze.

Der logische Positivismus geht also davon aus, dass letztlich jedes Phänomen auf der Grundlage von Beobachtungen und rationaler Folgerung analysierbar ist. In einem zweiten Schritt folgt daraus häufig die weitergehende Annahme, dass jedes Phänomen auf seine naturwissenschaftlichen, sprich physikalischen Grundlagen rückführbar ist. Diese Annahme basiert auf einer spezifischen Form des Reduktionismus, d. h. auf dem Glauben, dass ein Phänomen, dass auf der analytischen Ebene A beobachtbar, aber nicht erklärbar ist, auf einer anderen, tiefer gelegenen analytischen Ebene B abbildbar und erklärbar wird. Das Phänomen ist nicht mehr, als die Summe seiner Teile. So lässt sich beispielsweise eine körperliche Reaktion durch ein Verständnis bestimmter Organe verstehen, oder ein spezifisches Organ durch Analysen auf zellulärer Ebene usw. Es sei an dieser Stelle angemerkt, dass diese Position nicht erst im logischen Positivismus entwickelt wird. 
Schon die Cellularpathologie Rudolf Virchows hatte Mitte des 19. Jahrhunderts diese Form der Reduktion überzeugend demonstriert. (Virchow 1858) Der logische Positivismus abstrahiert sie von konkreten Fällen und erhebt sie zum allgemeinen Prinzip. Diese Form der Reduktion ist bis heute für die Naturwissenschaften prägend. Erkenntnis sei nur möglich, so die Denker des Wiener Kreises, wenn man diesen Grundsätzen folge. Wissen, das abseits der wissenschaftlichen Methode generiert werde, habe keine Gültigkeit. $^{2}$

Auch der frühen Kulturanthropologie war diese Perspektive keineswegs fremd. Franz Boas betrieb seine Studien zu körperlichen und geistigen Adaptionen an die neuen Lebensbedingungen von Immigranten in den Vereinigten Staaten oder Lautverschiebungen bei den Inuit mit genau diesem Anspruch. (z. B. Boas 1889) Und auch die Ethnographien im Stile eines Bronislaw Malinowski wurden lange Zeit als Vertextlichungen systematischer Beobachtung und damit auch als objektive Repräsentation von Welt verstanden. (z. B. Malinowski 1920) Bis in die 1950er Jahre hinein trat die Kultur- und Sozialanthropologie noch überwiegend als positivistische Wissenschaft auf. Margaret Mead, Gregory Bateson oder Edward T. Hall sind prominente Vertreterinnen und Vertreter dieser Phase (vgl. Mead 1961), die mit der Debatte zwischen Talcott Parsons und Alfred Kroeber über den Kulturbegriff (Parsons/Kroeber 1958) und dieser Debatte folgend der zumindest für die amerikanische Kulturanthropologie wegweisenden semiotischen Bestimmung von Kultur durch Clifford Geertz, zu einem Ende kam. (Geertz 1973) Einher damit ging der Aufstieg des Sozialkonstruktivismus in der Kulturanthropologie und vielen Bereichen der Sozialwissenschaften. Seit dieser Wende hat die Bezeichnung >Positivist` lange Zeit eher als Schmähung oder Schimpfwort gegolten und den so Bezeichneten eine mangelnde Reflektionsleistung und analytische Fähigkeit unterstellt. Eine solche Nutzung des Konzepts war allerdings zu keinem Zeitpunkt besonders höflich oder besonders hilfreich. Hinzukommt, dass zunehmend realistische Positionen seit dem Niedergang klassischer sozialkonstruktivistischer Ansätze in den 1990er Jahren eine Renaissance erleben. Diese Ansätze sind von den späten positivistischen Denkansätzen keineswegs so weit entfernt, wie oft angenommen wird. (z. B. Barad 1999) Vor allem die wichtige Rolle, die der materielle Körper in seiner physischen Umwelt für Mead, Bate-

2 Diese Denkweise wird auch als Szientismus bezeichnet. 
son, Hall und andere in der Analyse von menschlichem Zusammenleben gespielt hat, wird seit einiger Zeit am Schnittfeld von Sozial- und Kulturanthropologie und Science and Technology Studies wieder aufgegriffen. Dies ist vor allem feministischen Strömungen in der amerikanischen Kulturanthropologie (vgl. hierzu etwa die Arbeiten von Donna Haraway, Emily Martin oder Rayna Rapp, die in anderen Kapiteln vorgestellt werden) und stark praxistheoretisch orientierten Ansätzen in der britischen Sozialanthropologie geschuldet (vgl. hierzu Maurice Bloch oder Tim Ingold). ${ }^{3}$

In seiner Reinform wurde der logische Positivismus in der deutschsprachigen Philosophie mit der Auflösung des Wiener Kreises in den späten 1930er Jahren weitgehend aufgegeben. Danach spielte er vor allem in der amerikanischen analytischen Philosophie eine Rolle. Der kanadische Wissenschaftshistoriker und -philosoph Ian Hacking fasst die Grundposition der Positivisten wie folgt zusammen (Hacking 1983: 4-5):

- Beobachtung kann klar von Theorie getrennt werden;

- Wissen wächst kumulativ;

- Wissenschaft als Ganzes hat eine deduktive Struktur;

- Terminologie ist oder sollte präzise sein;

- alle Wissenschaften gemeinsam bilden eine Einheit und

- es ist möglich zwischen dem Inhalt einer Entdeckung und dem Kontext seiner Rechtfertigung zu unterscheiden.

Diese Zusammenfassung macht deutlich, dass die Grundsätze des logischen Positivismus heutzutage keineswegs obsolet sind. In vielen Bereichen der empirischen Wissenschaften, sowohl in den Natur-, als auch den Sozialwissenschaften, wird weitgehend selbstverständlich davon ausgegangen, dass Wissenschaft diesen Grundsätzen folgt und folgen sollte. Diese Grundsätze entsprechen auch weitgehend dem Bild von Wissenschaft, wie es im öffentlichen und medialen Diskurs präsent ist. Dabei ist zu beachten, dass diese Grundsätze zu Zeiten ihrer Entstehung sowohl deskriptiv wie normativ gedacht waren, d. h. sie beschrieben nicht nur, wie Erkenntnisgewinn durch

3 Letztere haben die Verschiebung in der Anthropologie hin zu Analysen von Bedeutungsproduktion, die die amerikanische Kulturanthropologie mit Geertz vollzog, nie wirklich mitgetragen. 
Wissenschaft von statten ging, sondern auch, wie Wissenschaftshandeln sein sollte. Rudolf Carnap war der festen Überzeugung, dass die Unterscheidung zwischen dem Inhalt einer Entdeckung und dem Kontext seiner Rechtfertigung klar voneinander zu trennen seien. Die Untersuchung des Kontexts der Rechtfertigung, d. h. die Umstände unter denen eine Entdeckung gemacht wurde, komme dabei den Geschichtswissenschaften zu und habe in der Wissenschaftsphilosophie lediglich illustrierenden und anekdotischen Wert. Der Inhalt der Entdeckung jedoch könne mittels des gerade geschilderten Verifikationsansatzes auf Wahrheit untersucht werden. Diese glasklare Trennung stieß schon früh auf Skepsis - nicht zuletzt bei Karl Popper, der dieser Sicht seinen kritischen Rationalismus entgegensetzte.

\section{KRITISCHER RATIONALISMUS UND FALSIFIKATION}

Karl Popper (1902-1994), in Wien geborener österreichisch-britischer Philosoph, wird häufig fälschlicher Weise dem Positivismus des Wiener Kreises zugerechnet. Zwar entspringt seine Argumentationslinie den Diskussionen um den Wiener Kreis. Auch teilt er mit der Carnapschen Position, dass Wissenschaft eine Einheit bildet, die sich letztlich in der Rückführbarkeit von Wissen auf eine universelle, wissenschaftliche Methode begründet. Popper selber wurde jedoch zu den Sitzungen des Kreises nicht eingeladen ${ }^{4}$ und entwickelte in seinem erkenntnistheoretischen Hauptwerk »Logik der Forschung« (Popper 1935; Popper 1959) eine Position, die in wichtigen Aspekten von den Überlegungen des logischen Positivismus abweicht.

Während der logische Positivismus des Wiener Kreises davon ausging, dass Aussagen durch Empirie und rationales Folgern verifiziert werden könnten, drehte Popper diese Sicht um. Verifizierung beruhe auf der Vorstellung der Möglichkeit induktiven Arbeitens, d. h. es werde von theoriefreien Beobachtungen ausgehend abstrahiert und theoretisiert. Da aber eine theoriefreie Beobachtung gänzlich unmöglich sei, also Theorie immer schon in Beobachtung enthalten sei, mache die Verifizierung von Theorie

4 Dies wohl hauptsächlich, weil Moritz Schlick sich von Poppers Art sowie seinem Gedankengut unangenehm berührt fühlte. 
durch Beobachtung keinen Sinn. Vielmehr stelle Wissenschaft Hypothesen auf, d. h. Verbindungen von Theorie und Empirie, die dann mittels Vernunft und wissenschaftlicher Methode kritisch hinterfragt werden müssten: daher kritischer Rationalismus. Hypothesen würden nicht durch Beobachtung verifiziert, sondern besäßen - einmal aufgestellt - so lange Gültigkeit bis sie widerlegt würden. Popper stellte dem Verifizierungsprinzip des logischen Positivismus sein Konzept der Falsifikation gegenüber. Dieses wird am verständlichsten, wenn man sich vor Augen hält, dass Popper sich mit behavioristischen, psychologischen Lerntheorien beschäftigt hatte. Er ging davon aus, dass der Mensch aktiv Erwartungen formuliert, die durch alltägliche Praxis, d. h. Versuch und Irrtum, erfüllt oder enttäuscht würden. Erkenntnistheoretisch gewendet und auf Wissenschaft angewandt, ergab sich daraus die Überlegung, dass Wissenschaft zunächst Hypothesen generiert und diese dann zu widerlegen sucht. Popper schrieb:

»Those among our theories which turn out to be highly resistant to criticism, and which appear to us at a certain moment of time to be better approximations of truth than other known theories, may be described, together with the reports of their tests, as >the science of that time. Since none of them can be positively justified, it is essentially their critical and progressive character - the fact that we can argue about their claim to solve our problems better than their competitors - which constitutes the rationality of science.« (Popper 1963: XII)

Wissenschaft besteht also laut Popper im Kern aus der kontinuierlichen Überprüfung von Hypothesen. Grundlage von Wissensproduktion ist damit nicht der Empirismus, d. h. der Versuch, Theorie durch empirische Befunde zu verifizieren, der den Wiener Kreis gekennzeichnet hatte, sondern ein kritischer Rationalismus. Dabei bildet Popper eine Figur des Übergangs zwischen den flat formalities ${ }^{5}$ des Wiener Kreises (Hacking 1983: 5) und den historisch fundierten Studien eines Thomas Kuhn und des nachfolgenden Historizismus insgesamt. Popper glaubte in positivistischer Manier an linearen Fortschritt, an eine Annäherung von Repräsentationen an Welt

5 Mit dem Begriff der flat formalities verweist Ian Hacking auf die eng formale und damit fast schon mechanische Anwendung von Logik als einzigem Mittel des philosophischen und damit wissenschaftlichen Erkenntnisgewinns durch die Vertreter des Wiener Kreises. 
und von wissenschaftlichen Aussagen an Wahrheit. Er war allerdings nicht überzeugt davon, dass der Wahrheitsgehalt von Aussagen positiv gerechtfertigt werden könne. Hypothesen könnten immer nur wieder überprüft werden. Dies ist nicht zu verwechseln mit einem wie auch immer gearteten Relativismus oder Nihilismus. Popper nahm an, dass wissenschaftliche Methode uns der Wahrheit näher bringt und dass unser Wissen wächst, auch wenn wir niemals irgendetwas mit letzter Sicherheit wissen können. Dies wird anschaulich in der folgenden Replik Poppers auf die Kritik Thomas Kuhns, der ihn als Relativisten bezeichnet hatte:

$» \mathrm{I}$ should like just to indicate briefly why I am not a relativist: I do believe in an absolute or objective truth, in Tarski's sense (although I am, of course, not an absolutist in the sense of thinking that I or anybody else has the truth in his pocket). I do not doubt that this is one of the points on which we are most deeply divided; and it is a logical point. I do admit that at any moment we are prisoners caught in the framework of our theories; our expectations; our past experiences; language. But we are prisoners in a Pickwickian sense: if we try, we can break out of our framework at any time. Admittedly, we shall find ourselves again in a framework, but it will be a better and roomier one; and we can at any moment break out of it again." (Popper 1970: 56)

Auch wenn Popper in den hitzigen Diskussionen der 1960er und frühen 1970er Jahre den Wert historischer, soziologischer und psychologischer Argumentation für zentrale Fragen der Wissenschaftstheorie als gering erklärte (vgl. Lakatos/Musgrave 1970), so begann er doch in seiner Wissenschaftstheorie, die enge, unausweichliche und formale Verschränkung von Beobachtung und Theorie, die der Wiener Kreis postuliert hatte, aufzuweichen. Ebenfalls stand er dem Projekt einer neutralen und universellen Beobachtungssprache skeptisch gegenüber. Er ging davon aus, dass der einzelne Wissenschaftler notwendig immer durch Interessen geleitet sei, d. h. Einzelergebnisse immer einen partikularen Status hätten. Dies hieß für ihn jedoch nicht, dass Wissenschaft als Ganzes lediglich partikulare oder subjektiv geprägte Befunde produziere. Er redete nicht einem Relativismus das Wort, da er davon ausging, dass Wissenschaft als Ganzes diese partikularen Interessen auf quasi evolutionäre Weise über einen zeitlichen Verlauf hinweg ausselektieren würde. $\mathrm{Zu}$ einiger Berühmtheit gelangte seine gemäßigt positivistische Position in der als Positivismusstreit 
berühmt gewordenen Debatte mit Theodor W. Adorno, in der verhandelt wurde, welche Erkenntnismöglichkeiten und -notwendigkeiten modernen Gesellschaften in der zweiten Hälfte des 20. Jahrhunderts zur Verfügung stünden. (WiSSENSCHAFTSSOZIOLOGIE)

\section{PaRAdigmenWeChSEl Und Historizismus}

Der dritte hier vorgestellte Wissenschaftstheoretiker des 20. Jahrhunderts, Thomas Kuhn (1922-1996) in Cincinnatti als Sohn jüdischer Eltern geboren und zunächst in Harvard als Physiker ausgebildet, vertrat explizit nicht die logische Position eines Carnap oder Popper, sondern eine historische Argumentationslinie: daher wird die von ihm propagierte Variante der Wissenschaftstheorie auch als Historizismus bezeichnet. Er kritisierte zunächst Popper dafür, dass dieser seine allgemeinen Überlegungen zum kritischen Rationalismus aus der Analyse von ungewöhnlichen Beispielen entwickelt hatte. Ungewöhnlich seien diese Beispiele aus Sicht Kuhns insofern, als dass sie Perioden des Umbruchs darstellten, in denen bisherige Grundannahmen scheinbar plötzlich verworfen und durch neue ersetzt würden. Klassischer Weise hatten sich positivistische und rationalistische Analysen an den großen Debatten und Umbrüchen in der theoretischen Physik abgearbeitet, beispielsweise dem Aufkommen der Quantenmechanik zur Beschreibung atomarer und subatomarer Vorgänge.

In dieser Kritik am Fokus der frühen wissenschaftstheoretischen Studien auf ungewöhnliche Phasen sind bereits die beiden zentralen Konzepte Kuhns angelegt: (1) Er unterscheidet zwischen Normalwissenschaft und wissenschaftlichen Revolutionen: Normalwissenschaft bezeichnet den wissenschaftlichen Arbeitsalltag, der im Wesentlichen aus dem routinisierten Lösen von Rätseln und Problemen besteht (Engl. puzzle-solving). In diesem Arbeitsalltag werden in der Regel keine theoretischen Grundannahmen in Frage gestellt. Vielmehr geschieht diese Arbeit innerhalb eines breit anerkannten theoretischen Rahmens. (2) Diesen Rahmen hat Kuhn in seinen frühen Arbeiten als Paradigma bezeichnet: »A paradigm is what members of a scientific community, and they alone, share.« (Kuhn 1977) Paradigmenwechsel, so schreibt Kuhn in seinem berühmtesten Werk »The structure of scientific revolutions« (Kuhn 1970), vollziehen sich durch wissenschaftliche Revolutionen, in denen inkommensurable Paradigmen aufein- 
ander treffen, d. h. Denkrahmen, die nicht miteinander vereinbar sind und daher nicht zur selben Zeit am selben Ort sein können.

Ein berühmtes Beispiel sind die Mechanik Newtons und Einsteins. Beide lassen sich in der Frage der Absolutheit des Raumes bzw. des Äthers nicht miteinander vereinbaren. $\mathrm{Zu}$ einem Paradigmenwechsel kommt es dann, wenn empirische Befunde nicht mehr innerhalb des etablierten Paradigmas erklärt werden können und ad-hoc Theorien nicht genügen, um die Abweichungen zu erklären. Kuhn vergleicht diesen Prozess mit einem Gestaltwechsel, wie er beispielsweise in dem bekannten Ente-Kaninchen Vexierbild auftritt (siehe Abb. 1).

\section{Abbildung 1: Ente-Kaninchen Vexierbild}

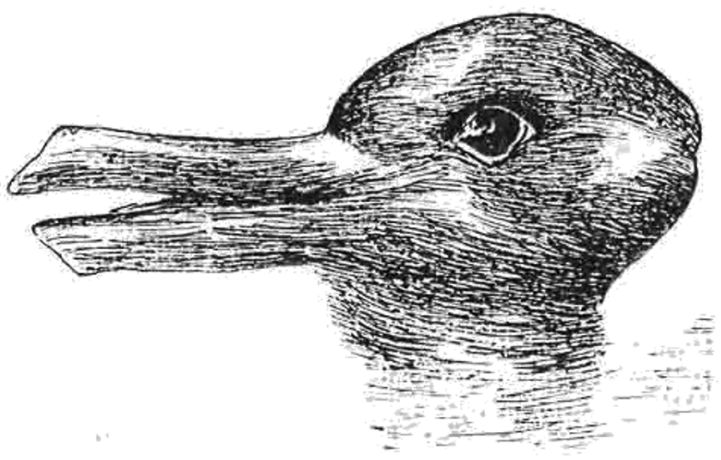

Quelle: Jastrow, J. (1899). The mind's eye. Popular Science Monthly, 54, 299-312.

Wir können in dieser Abbildung gegebenenfalls mit entsprechender Anleitung einen Hasen oder eine Ente sehen; nur niemals beide gleichzeitig. Kuhn entlehnt dieses Konzept der Inkommensurabilität von Paradigmen aus der Mathematik und entwickelt es zeitgleich mit Paul Feyerabend, der allerdings mit seiner globalen Inkommensurabilität radikaler denkt, als Kuhn dies mit seiner Form der lokalen Inkommensurabilität tut. (Vgl. Feyerabend 1975) ${ }^{6}$ Der Prozess des Lernens, als dessen Ergebnis dann

6 Zur Erläuterung: Kuhn begrenzte sein Konzept der Inkommensurabilität auf verschiedene Wissensschaftsgemeinden oder Forschergruppen, die in einer spezifi- 
entweder ein Hase oder eine Ente zu sehen ist, entspricht für Kuhn der wissenschaftlichen Disziplinierung. Er strukturiert Denken, Erfahrung und Handeln der Forschenden, ohne dass diese sich notwendiger Weise dessen bewusst sind. Der hierdurch entstehende blinde Fleck spielt in der Forschung der Science and Technology Studies eine wichtige, und nicht unproblematische Rolle, wie viele der Beiträge in diesem Band immer wieder vor Augen führen.

Kuhns Rolle in der Entwicklung der Science and Technology Studies ist ambivalent. Einerseits hat seine Idee des Paradigmenwechsels eine absolut zentrale Rolle in der Entwicklung der empirischen Wissenschaftsforschung gespielt. Dieses Konzept hat zunächst historische, dann aber auch sozialwissenschaftliche und ethnographische Studien von Wissenschaft als Praxis zu legitimieren geholfen. Kuhns Beitrag zum Feld der Science and Technology Studies kann also gar nicht hoch genug eingeschätzt werden. Andererseits jedoch hat die empirische Wissenschaftsforschung Kuhns Beiträge in fast allen Belangen so rigoros überarbeitet, dass sein Konzept des Paradigmenwechsels heutzutage lediglich eine wissenschaftshistorische Präsenz und Bedeutung besitzt - auch wenn es in der breiten Öffentlichkeit nach wie vor als Klassiker und wohl am meisten rezipiertes Konzept der Wissenschaftstheorie und -geschichte überhaupt gelten kann.

Die kritische Lesart von Kuhns Texten ist allerdings kein Phänomen der letzten zwanzig Jahre, sondern begann bereits 1962 mit Erscheinen seiner »Scientific Revolutions«. Erstens wurde die Unterscheidung zwischen Normalwissenschaft und wissenschaftlicher Revolution von seinen Kritikern angezweifelt und letztlich aufgelöst. Das Beharren auf Theorien und ihre Ausweitung würden immer Hand in Hand gehen, und es mache weder deskriptiv noch normativ Sinn, zwischen normal und revolutionär so rigoros zu unterscheiden, wie Kuhn dies tue. Diese Argumente brachten vor allem John W.N. Watkins, Popper und Feyerabend vor (Watkins 1970, Feyerabend 1970, Popper 1970). Stephen Toulmin verabschiedete das Konzept der wissenschaftlichen Revolution endgültig, in dem er mittels einer Analogie aus den Politikwissenschaften deutlich machte, dass das Konzept der Revolution immer nur ein Platzhalter für etwas sei, was im ersten Moment

schen Frage konträre Paradigmen entwickeln. Feyerabend war der Überzeugung, dass es unabhängig von lokalen Bedingungen, global inkommensurable Paradigmen oder Denkstile gäbe. 
nicht fassbar, bei genauerer Analyse aber durchaus verständlich und strukturierbar sei (Toulmin 1970). Kuhns Konzept der wissenschaftlichen Revolution sei also hauptsächlich der Art der Analyse geschuldet, die nicht hoch genug auflösend bzw. detailliert vorgegangen sei. Zweitens wurde das Konzept des Paradigmas von vielen als unscharf kritisiert und, wenn man die Idee überhaupt gut hieß, häufig auf Überlegungen des britischen Wissenschaftsphilosophen Robin G. Collingwood zurückgeführt, der sein sehr ähnliches Konzept der absolute presuppositions bereits 1940 entwickelt hatte (Collingwood 1940/1972). Kuhn selbst sagte nicht lange vor seinem Tod: »Paradigm was a perfectly good word, until I messed it up.« (Kuhn 2000) Drittens wurde von Anfang an das Konzept des Paradigmenwechsels als Abfolge von inkommensurablen Paradigmen in Frage gestellt. Ohne die Leitunterscheidung zwischen normaler und revolutionärer Wissenschaft mache der schlagartige Wechsel wenig Sinn und so wurde dem abrupten Paradigmenwechsel bereits früh das Konzept eines langsameren und vielschichtigeren, wenn nicht sogar kontinuierlichen Übergangs gegenübergestellt, der nur durch sehr selektive Historisierung als schlagartiger Wechsel beschreibbar sei.

Trotz dieser frühen elementaren Kritik hat sich das Konzept des Paradigmenwechsels zu einem Selbstläufer entwickelt und enorme Karriere gemacht - eben auch in den frühen empirischen Wissenschaftsforschungsstudien. Allerdings wurde in den detaillierten Analysen von wissenschaftlichem Alltag schnell deutlich, dass Kuhns Analyse wissenschaftliche Praxis lediglich in sehr idealistischer Weise schilderte und begreifbar machte nicht aber in der Art und Weise, wie sie tatsächlich von statten ging und geht. Dieser idealistische Analysestil mag der Zeit und der Notwendigkeit geschuldet sein, eine Anschlussfähigkeit in die Wissenschaftsphilosophie herzustellen. Er rührt aber auch von der Tatsache her, dass Kuhn, der selbst als Physiker in Harvard begonnen hatte, bevor er in die Geschichte und Philosophie der Wissenschaft wechselte, vor allem die großen Konzeptentwicklungen und -brüche in der Physik vor Augen hatte. Diese wurden aber historisch immer als abstrakte Auseinandersetzungen über Theorie kolportiert und nicht als situierte Alltage von tatsächlichen Physikerinnen und Physikern; eine ideengeschichtliche oder eben idealistische Lesart von Wissenschaftstheorie lag also auch im empirischen Material Kuhns begründet. Gerade diese idealistische Art des Paradigmakonzepts war es jedoch, die diejenigen, die ethnographische Analysen von Wissensproduktion und 
Technologieentwicklung als spezifische, situierte Praxis durchführten, anregten, alternative Konzepte zu entwerfen, um die verschiedenen Facetten von tatsächlichem Wissenschaftsalltag in den Blick zu bekommen. Konzepte wie Plattform, Experimentalsystem oder Assemblage sind nur einige Begriffe, die den Alltag von Wissensproduktion weniger idealistisch und stärker gebunden an Technologie, Episteme oder Macht-Wissen verstehen. (Ong/Collier 2005; Cambrosio et al. 2009; Rheinberger 1997; LABORSTUDIEN)

\section{LUdWIK Fleck: Denkstile und DenkKollektive}

Der polnische Mediziner und Mikrobiologe Ludwik Fleck (1896-1961) arbeitete in den 1920er und 1930er Jahren in der Abteilung Innere Medizin des Allgemeinen Krankenhauses von Lemberg (heutige Ukraine; ukrainisch: Lwiw, polnisch: Lwów) und in verschiedenen bakteriologischen Instituten, wo er sich vor allem mit der Diagnose und Bekämpfung von Infektionskrankheiten wie Typhus und Syphilis beschäftigte. Lemberg wurde 1939 sowjetisch besetzt und kurze Zeit später von deutschen Truppen eingenommen. Fleck, seine Frau und sein Sohn wurden wegen ihrer jüdischen Herkunft im jüdischen Ghetto von Lemberg interniert. Als ausgewiesener Experte in der Impfstoffentwicklung wurde Fleck von den Nazis gezwungen, seine Arbeit zunächst in Lemberg, später in den Konzentrationslagern Auschwitz und Buchenwald fortzusetzen. Angeblich hat Fleck die SS mit wirkungslosen, die Mithäftlinge aber mit wirksamen Impfseren versorgt. ${ }^{7}$ Fleck, seine Frau und sein Sohn überlebten den Zweiten Weltkrieg. Fleck setzte nach dem Krieg seine Karriere fort, emigrierte nach Herzinfarkt und Krebsdiagnose mit seiner Frau zu seinem Sohn nach Israel und starb dort 1961.

Bereits 1935 publizierte Fleck auf Deutsch sein Hauptwerk »Die Entstehung und Entwicklung einer wissenschaftlichen Tatsache« (Fleck 1935/1980). In der polnischen Schule der Wissenschaftstheorie fand dieses Werk zunächst wenig Beachtung (Löwy 1990). Kuhn erwähnte es in seinem Vorwort zu »Scientific Revolutions« und verhalf ihm damit posthum

7 Zur Debatte über Flecks Studien in den Konzentrationslagern, siehe Fehr et al. (2008) und Weisz (2010). 
zu internationaler Sichtbarkeit. Trotzdem wurde es erst 1979 ins Englische übersetzt mit dem Titel »Genesis and development of a scientific fact» (Fleck 1979). Dieses Buch, das wohl eher einen Entwurf denn eine voll entwickelte Theorie darstellt, ist insofern Urgestein der Science and Technology Studies, als es als erstes Werk den Produktionsprozess von Erkenntnis als Praxis und nicht als logische Struktur in den Mittelpunkt wissenschaftlicher Überlegungen stellt. Fleck schreibt nicht aus dem abstrakten Interesse des Philosophen am Erkenntnisprozess heraus, sondern vor allem als medizinischer und mikrobiologischer Praktiker. Er reflektiert seinen eigenen Arbeitsalltag, seine Ausbildung und damit das Feld, in dem er arbeitet, und wendet diese Reflektionsleistung präzise in eine wissenschaftstheoretische Glanzleistung, die sich abseits des damals gängigen philosophischen Vokabulars bewegt und die bis heute prägend ist. Diese Form des Arbeitens, die man heutzutage vielleicht als para-ethnographisch bezeichnen würde (Marcus 2008), ist in den Science and Technology Studies nicht ungewöhnlich. Im Gegenteil: das Forschungsfeld hat bis heute immer wieder in entscheidenden Momenten seiner Entwicklung von Wissenschaftlerinnen (seltener Wissenschaftlern) mit Doppelausbildung profitiert. Gerade das Schnittfeld von feministischer Anthropologie und Wissenschaftsforschung hat sich hier als fruchtbar erwiesen: Donna Haraway, Karen Barad, Margaret Lock sind prominente Vertreterinnen dieser Richtung mit ursprünglich naturwissenschaftlicher Ausbildung. Allerdings muss angemerkt werden, dass die Kenntnis des zu untersuchenden Feldes als quasi »Eingeborene« nur dann von Vorteil ist, wenn die betroffene Person die unterschiedlichen Perspektiven klar zu trennen und analytisch zu ihrem Vorteil zu wenden versteht. Fleck jedenfalls - wie auch den erwähnten feministischen Anthropologinnen - ist dies hervorragend gelungen und er kann mit Fug und Recht als einer der ersten praxistheoretischen Wissenschaftsforscher gelten. Sein Hauptwerk, wohlwollend gelesen, hat bis heute wenig an Aktualität eingebüßt. (Vgl. Löwy 2008)

Fleck stellt gleich zu Beginn seines Werks deutlich heraus, wo er das Hauptproblem der philosophischen Wissenschaftstheorie seiner Zeit sieht, d. h. vor allem das Hauptproblem des Wiener Kreises. Die Wissenschaftstheorie untersuche den Erkenntnisprozess als rationalen, individuellen Akt und ziehe immer wieder Beispiele aus der Vergangenheit statt der aktuellen wissenschaftlichen Praxis heran, d. h. »alte Tatsachen«. Deren Entstehungsprozess sei nicht mehr sichtbar und nachvollziehbar: 
»[Die Tatsache] ist uns selbstverständlich geworden und sie dünkt uns fast gar kein Wissen mehr, wir fühlen nicht mehr unsere Aktivität bei diesem Erkenntnisakte, nur unsere vollständige Passivität gegenüber einer von uns unabhängigen Macht, die wir Existenz oder Realität nennen.« (Fleck 1935: 1)

Fleck ging es also darum, die Entstehung und Entwicklung von Tatsachen anders zu erklären, als dies die Philosophen und Historiker seiner Zeit zu tun pflegten. Fleck arbeitete hauptsächlich mit Syphilis als Beispiel, speziell der Entstehung und Entwicklung des Nachweises von Syphiliserregern, der sogenannten Wassermannreaktion, benannt nach August von Wassermann, der diesen Test im frühen 20. Jahrhundert entwickelte. Fleck legte Wert auf den Produktionsprozess von Tatsachen aus historischer, psychologischer und soziologischer Sicht. Die gängige Analyse erkenntnistheoretischer Entscheidungen - welcher Theorie folge ich, wie baue ich ein Experiment auf? - berücksichtige, laut Fleck, viel zu wenig ihre kulturhistorische Bedingtheit: »Es besteht eine stilmäßige Bindung aller - oder vieler - Begriffe einer Epoche, die auf ihrer gegenseitigen Beeinflussung beruht. Deshalb kann man von einem Denkstil sprechen, der den Stil jedes Begriffes bestimmt.« (Fleck 1935: 15) Denkstil bezeichnet ein denkgeschichtlich geprägtes Gefüge von Konventionen. Diese Konventionen binden dann jeweils Wissensproduktion kultur- wie erkenntnisgeschichtlich und verstellen den einzelnen Forschenden die Möglichkeit der freien, rationalen Wahl. Im Gegensatz zum Konzept des Paradigmas ist das Konzept des Denkstils situiert in einer spezifischen Epoche, einem wissenschaftlichen Kollektiv und vor allem in wissenschaftlicher Praxis.

Anders als alle seine Zeitgenossen (und viele Wissenschaftler_innen bis heute) betonte Fleck immer wieder die soziale Komponente dieser Konventionen und des Erkenntnisprozesses als Praxis. Zunächst erklärte er, dass Denkstile immer an ein Denkkollektiv gebunden seien: »Ein Denkkollektiv ist immer dann vorhanden, wenn zwei oder mehrere Menschen Gedanken austauschen. [...] Persönliche Heldentaten bleiben nur dann bestehen, wenn sie Suggestivwirkung ausüben.« (ebd.: 60) Auch wenn Fleck nicht notwendiger Weise von den anthropologischen Denkerinnen und Denkern 
seiner Zeit wusste ${ }^{8}$, so hat er doch eine im Kern beinahe kultur- und sozialanthropologische Perspektive vertreten, die später auch immer wieder von anthropologisch Forschenden aufgegriffen wurde. Marilyn Strathern, eine der einflussreichsten britischen Anthropologinnen der zweiten Hälfte des 20. Jahrhunderts, spricht 70 Jahre später davon, dass Wissen immer nur dann interessant sei, »if others can invent around it« (Strathern 2002) und fasst damit ebenfalls genau die soziale Komponente von Wissensproduktion, die auch für Fleck schon zentral steht. (in ähnlicher Form auch bei Mary Douglas; KLASSIFIKATIONEN) Fleck schreibt bereits 1935: „Das Erkennen stellt die am stärksten sozial bedingte Tätigkeit des Menschen vor und die Erkenntnis ist das soziale Gebilde [schlechthin]. [...] Wessen Gedanke ist es, der weiter kreist? Ein Kollektivgedanke eben, einer, der keinem Individuum angehört.« Und weiter:

»Jede Erkenntnistheorie, die diese soziologische Bedingtheit allen Erkennens nicht grundsätzlich und einzelhaft ins Kalkül stellt, ist Spielerei. Wer aber die soziale Bedingtheit für ein malum necessarium, für eine leider existierende menschliche Unzulänglichkeit ansieht, die zu bekämpfen Pflicht ist, verkennt, dass ohne soziale Bedingtheit überhaupt kein Erkennen möglich sei, ja, dass das Wort `Erkennen` nur im Zusammenhang mit einem Denkkollektiv Bedeutung erhalte.« (Fleck 1935: 59)

Ungewöhnlicher für seine als für die heutige Zeit mutet auch folgender Vergleich an:

»Das Individuum ist dem einzelnen Fußballspieler vergleichbar, das Denkkollektiv der auf Zusammenarbeit eingedrillten Fußballmannschaft, das Erkennen dem Spielverlaufe. Vermag und darf man diesen Verlauf nur vom Standpunkte einzelner Fußstöße aus untersuchen? Man verlöre allen Sinn des Spieles!« (Fleck 1935: 62)

Wichtiger Bezugspunkt für dieses Gedankengut ist für Fleck wohl weniger die Anthropologie als die Soziologie und die (Wissenschafts-)Philosophie seiner Zeit. Er zitiert beispielsweise einen der Gründerväter der eu-

8 Untersuchungen, inwieweit Fleck von anthropologischen Zeitgenossen beeinflusst war, und wer noch in die Genealogie seines Denkens gehört, finden sich bei Gonzales et al. 1995. 
ropäischen Soziologie, den polnischen Juristen Ludwig Gumplowicz, der bereits 1905 schrieb:

»Der größte Irrtum der individualistischen Psychologie ist die Annahme, der Mensch denke. Aus diesem Irrtum ergibt sich dann das ewige Suchen der Quelle des Denkens im Individuum und der Ursachen, warum er so und nicht anders denke, woran dann die Theologen und Philosophen Betrachtungen darüber knüpfen oder gar Ratschläge erteilen, wie der Mensch denken solle. Es ist dies eine Kette von Irrtümern. Denn erstens, was im Menschen denkt, das ist gar nicht er, sondern seine soziale Gemeinschaft. Die Quelle seines Denkens liegt gar nicht in ihm, sondern in der sozialen Umwelt, in der er lebt, in der sozialen Atmosphäre, in der er atmet, und er kann nicht anders denken als so, wie es aus den in seinem Hirn sich konzentrierenden Einflüssen der ihn umgebenden sozialen Umwelt mit Notwendigkeit sich ergibt.« (Gumplowicz 1905 in Fleck: 63 ff.)

Fleck scheint von dieser Argumentationslinie stark beeindruckt gewesen zu sein und wendet sie gegen den Wiener Kreis, das dominante Denkkollektiv seiner Zeit in der Wissenschaftstheorie:

»Einen [...] ebenfalls sehr charakteristischen Fehler begehen die philosophierenden Naturforscher. Sie wissen, dass es keine seinzig und allein objektiven Merkmale und Verhältnisse gebe, sondern nur Relationen in Bezug auf ein mehr oder weniger willkürliches Bezugssystem. Aber sie begehen ihrerseits den Fehler, allzu großen Respekt vor Logik, eine Art religiöser Hochachtung vor logischem Schließen zu haben. Für diese naturwissenschaftlich gebildeten Erkenntnistheoretiker, z. B. des so genannten Wiener Kreises (Schlick, Carnap u.a.) ist menschliches Denken (wenigstens als Ideal, als Denken, wie es sein soll) ein Fixum, ein Absolutum - die empirische Tatsache dagegen das Relative. Umgekehrt sehen die angeführten humanistisch gebildeten Philosophen in der Tatsache das Fixum, im menschlichen Denken hingegen das Veränderliche. Charakteristisch, wie beide Parteien das Fixum in den ihnen fremden Bereich verlegen!« (Fleck 1935: 69)

Wie entsteht und entwickelt sich also nun eine wissenschaftliche Tatsache laut Fleck? Im chaotischen anfänglichen Denken zeigt sich zunächst was Fleck as Widerstandsaviso bezeichnet, d. h. eine erste Ahnung, dass etwas Signifikantes im Entstehen begriffen ist, das sich mit dem bisherigen Wissen nicht greifen und erklären lässt. Oft entsteht dieses Widerstandsaviso 
durch ein sich regen der »Realität«, die Widerstand leistet dagegen, auf eine bestimmte Art und Weise gedacht oder experimentell geformt $\mathrm{zu}$ werden. Es formt sich sodann ein bestimmter Denkzwang und »schließlich eine unmittelbar wahrzunehmende Gestalt«. Dieser Prozess der Erkenntnis ist erstens immer das Ergebnis denkgeschichtlicher Zusammenhänge und eines bestimmten Denkstils. "Vergangenheit lebt in übernommenen Begriffen, Problemfassungen, schulmäßiger Lehre, Institutionen, Sprache und alltäglichem Leben weiter.« Zweitens arbeitet wissenschaftliche Erkenntnis mittels der Erfahrung und des Experiments. »Kann Experiment als einfache Frage und Antwort gedeutet werden, so ist Erfahrung bereits als verwickelte Erzogenheit zu verstehen.« Hier nimmt Fleck sowohl den Foucaultschen medizinischen Blick als auch die materiell-semiotischen Koproduktionstheorien der feministischen Anthropologie vorweg. (STS UND POLITIK) Ebenfalls setzt er sich hier in drei wichtigen Punkten sowohl vom logischen Positivismus wie vom kritischen Rationalismus seiner Zeit ab:

Erstens verleiht er mit dem Konzept des Stils dem Erkenntnisprozess eine kultur- und sozialhistorische Tiefe und betont dabei auch die Rolle von Technologie und Materialität, die in der Wissenschaftsphilosophie bis dato keine Rolle gespielt hatten: »Tradition, Erziehung und Gewöhnung rufen eine Bereitschaft hervor für stilgemäßes, d. h. gerichtetes und begrenztes Empfinden und Handeln. Bis in der Frage die Antwort größtenteils vorgegliedert ist und man sich nur für ein Ja oder Nein oder für ein zahlenmäßiges Feststellen entscheiden muss. Bis Methoden und Apparate den größten Teil des Denkens für uns von selbst ausführen.«

Zweitens postuliert er jenseits von Verifikation und Falsfikation, dass der Zusammenhang von Theorie und Beobachtung vielmehr eine Frage von Plausibilisierung sei: »Wäre ein Forschungsexperiment klar, so wäre es überhaupt unnötig: denn um ein Experiment klar zu gestalten, muss man sein Ergebnis von vorneherein wissen, sonst kann man es nicht begrenzen und zielbewusst machen.«

Drittens merkt er die Koproduktion von Erkenntnis und Erkennenden an: »Das Erkennen verändert den Erkennenden, ihn an das Erkannte harmonisch anpassend, und dieser Umstand sichert die Harmonie innerhalb der herrschenden Meinung über die Entstehung der Erkenntnis. [....] Jede empirische Entdeckung kann also als Denkstilergänzung, Denkstilentwicklung oder Denkstilumwandlung aufgefaßt werden.« (alle vorangegangen Zitate, Fleck 1935: 120ff.) 
Ähnlich wie später Kuhn, geht Fleck von Theoriephasen aus. In den klassischen Phasen stehen die regelmäßigen Fälle im Vordergrund: Plausibilität, Abgeschlossenheit und Propagandafähigkeit von Theorien ist kennzeichnend. Mit zunehmender Spezialisierung nehmen die Knotenpunkte zu. Fleck unterscheidet in diesem Zusammenhang zwischen aktiven und passiven Kopplungen. Aktive Kopplungen bezeichnen die von den Forschenden gestalteten Kopplungen, die sich denkhistorisch, denkpsychologisch und denksozial erklären lassen. Passive Kopplungen ergeben sich zwangsweise im Forschungsprozess aus den sachlichen und materiellen, den »wirklichen« Verbindungen. Aktive und passive Kopplungen sind notwendiger Weise miteinander verschränkt. Je dichter verschränkt diese Kopplungen, desto geringer die Meinungsdifferenzen und desto stabiler ein Denkstil. Wenn die »Ausnahmen die regelmäßigen Fälle zu überwuchern« beginnen (Paul Ehrlich zitiert in Fleck: 42), nimmt die Beharrlichkeit von Meinungssystemen ab. Ein Widerstandsaviso tritt auf und der Prozess beginnt von vorn.

Wie nun soll man diesen Prozess untersuchen? Auch hier nimmt Fleck Vieles, was in der zweiten Hälfte des 20. Jahrhunderts entwickelt wird, bereits vorweg. Sein Plädoyer gilt einer vergleichenden Epistemologie als Sozialtheorie. Fleck argumentiert, dass sich in bestimmten Epochen und so auch in der Gegenwart, relativ stabile Gebilde aus Auffassungen, Denkstilen, Präideen usw. ausprägen. Fleck prägt den Begriff der Harmonie der Täuschungen, um zu beschreiben, wie diese verschiedenen Elemente derart ineinander greifen, dass sie in ihrer Gesamtheit nicht mehr als kontingentes Phänomen erscheinen. Sie verlieren scheinbar ihre ontologische Verhandelbarkeit und täuschen in ihrem harmonischen Zusammenwirken vor, dass die Welt - und das Wissen und Beforschen von Welt - immer nur so sein kann, wie es gerade ist. Aufgabe der Science and Technology Studies ist es nun, diese Harmonie der Täuschungen aufzulösen, d. h. für ein Wissensgebiet die verschiedenen Entwicklungslinien zunächst separat, dann in ihren Wechselwirkungen und Verbindungen nachzuzeichnen, ohne den Blick für die Entwicklung des gesamten Feldes zu verlieren. Gerade bei der Untersuchung aktueller Themen ist dies vor allem deswegen schwierig, weil die Harmonie der Täuschung die Untersuchenden schnell selbst hinter das Licht führt. Vor allem dann, wenn sie sich nur in einem Feld bewegen. 


\section{FAZIT}

Fleck, ganz Anthropologe, schlägt daher vor, diesen Harmonien der Täuschungen komparatistisch, d. h. vergleichend zu begegnen. Dadurch erführe man zum einen etwas über die spezifischen Ideen, also z. B. die Wassermannreaktion oder ein spezifisches Impfserum. Zum anderen lerne man aber über das Vergleichen und Erforschen der Zusammenhänge, die zur Harmonie der Täuschungen führen, viel über den Kontext in dem diese Harmonie sich zusammenfügt, d. h. über die Epoche, die Gesellschaft und das wissenschaftliche Milieu. Dies gilt natürlich im Besonderen für ethnographische Forschung in der eigenen Gesellschaft. Die vergleichende Analyse verschiedener Täuschungen ermöglicht es daher, den blinden Fleck einer Einzelperspektive zu verkleinern, den man in Studien der eigenen Epoche zwangsläufig ausprägt. Daher: Vergleichende Epistemologie als Sozial- und Kulturtheorie.' Denn die Science and Technology Studies, zumindest in ihrer sozialanthropologischen Ausprägung, wie sie in diesem Band vorgestellt werden, untersuchen die Entwicklungen von Wissenschaft und Technologie und ihre verschiedenen Anwendungsfelder nicht nur, um diese Entwicklungen selbst besser zu verstehen oder um ihre gesellschaftlichen Folgen im engeren Sinne abzuschätzen. Vielmehr verrät die vergleichende Ethnographie dieser verschiedenen Entwicklungen immer auch etwas über die Gesellschaft, in der diese Entwicklungen stattfinden. Sie verrät etwas über soziale Ordnungen, über die vielfältigen Verschränkungen von Natur und Kultur und über moralische Ordnungen und Kosmologien. (Herzfeld 1997) In Fleckscher Tradition stehend, versteht daher die sozialanthropologische Wissenschafts- und Technikforschung Wissen und Technologie nicht als etwas außerhalb von Kultur, das sich auf diese auswirkt, sondern als etwas zutiefst Menschliches, historisch wie kulturell Situiertes und in mannigfaltig ausdifferenzierter Praxis immer wieder Reproduziertes - und damit als zentrales Element ethnographischer Forschung.

9 Siehe vertiefend die exzellente Arbeit des US amerikanischen Medizinanthropologen und Wissenschaftshistorikers Allan Young »The Harmony of Illusions « zur Entstehung und Entwicklung von posttraumatischer Belastungsstörung bei US amerikanischen Vietnamkriegsveteranen (Young 1995). 


\section{LITERATUR}

Barad, Karen (1999): »Agential Realism. Feminist Interventions in Understanding Scientific Practices«. In: Mario Biagioli (Hg.), The Science Studies Reader, New York: Routledge, S 1-11.

Boas, Franz (1889): »On Alternating Sounds«. American Anthropologist 2,(1), S. 47-54.

Cambrosio, Alberto, Peter Keating, Pascalle Bourret, Phillipe Mustar, and Susan Rogers. (2009): »Genomic Platforms and Hybrid Formations«. In: Paul Atkinson/Peter Glasner/Margaret Lock (Hg.), Handbook of Genetics and Society. Mapping the New Genomic Era, London: Routledge.

Carnap, Rudolf (1934/1968): Logische Syntax der Sprache, Wien: Springer.

Collingwood, Robin G. (1940/1972) An Essay on Metaphysics, Chicago: H. Regnery Co

Feyerabend, Paul (1970): „Consolations for the Specialist«. In: Alan Musgrave/Imre Lakatos (Hg.), Criticism and the Growth of Knowledge, Cambridge: Cambridge University Press, S. 197-230.

Fleck, Ludwig (1935/1980): Entstehung und Entwicklung einer wissenschaftlichen Tatsache. Einführung in die Lehre vom Denkstil und Denkkollektiv. Mit einer Einleitung herausgegeben von Lothar Schäfer und Thomas Schnelle. (1935 Basel: Benno Schwabe \& Co.), Frankfurt/M.: Suhrkamp.

Fleck, Ludwik (1979): Genesis and Development of a Scientific Fact, Chicago: University of Chicago Press.

Geertz, Clifford (1973): The Interpretation of Cultures, New York: Basic Books.

Gonzales, R. J., Laura Nader, \& Ou:, C. J. (1995): »Between two Poles: Bronislaw Malinowski, Ludwik Fleck, and the Anthropology of Science«. [Anthropologie] Current Anthropology, 36(5), S. 866-869.

Hacking, Ian (1983): Representing and Intervening: Introductory Topics in the Philosophy of Natural Science, Cambridge: Cambridge University Press.

Herzfeld, Ma. (1997). Cultural Intimacy. Social Poetics in the Nation State. New York Routledge. 
Kuhn, Thomas (2000): The Road since Structure. Philosophical Essays 1970-1993, Chicago: Chicago University Press.

Kuhn, Thomas S. (1977): The Essential Tension: Selected Studies in Scientific Tradition and Change, Chicago: University of Chicago Press.

Kuhn, Thomas S. (1970): The Structure of Scientific Revolutions, Chicago: University of Chicago Press.

Lakatos, Imre, and Alan Musgrave, eds. Criticism and the Growth of Knowledge. Proceedings of the International Colloquium in the Philosophy of Science, London, 1965. dt.: Kritik und Erkenntnisfortschritt, Braunschweig 1973 ed. Vol. 4. Cambridge Cambridge University Press, 1970.

Löwy, Ilana (1990): The Polish School of Philosophy of Medicine : From Tytus Chalubinski (1820-1889) to Ludwik Fleck (1896-1961), Philosophy and Medicine, Dordrecht/Boston: Kluwer Academic.

Löwy, Ilana (2008): »Ways of seeing: Ludwik Fleck and Polish debates on the perception of reality, 1890-1947«. Studies in the History and Philosophy of Science 39, S. 375-383.

Malinowski, Bronislaw (1920): Argonauts of the Western Pacific, London: Routledge.

Marcus, George (2008): »The End(S) of Ethnography: Social/Cultural Anthropology's Signature Form of Producing Knowledge in Transition«. Culture Anthropology 23(1), S. 1-14.

Mead, Margaret (1961): »Anthropology among the Sciences«. American Anthropologist 63(3), S. 475-82.

Ong, Aihwa/Collier, Stephen J. (2005): Global Assemblages Technology, Politics, and Ethics as Anthropological Problems, Malden/MA: Blackwell Publishing.

Parsons, Talcott/Kroeber, Alfred L. (1958): »The Concepts of Culture and of Social Systems«. American Sociological Review 23, S. 582-83.

Popper, Karl R. (1935/2005): Die Logik der Forschung, 11. Ausg., Tübingen: Mohr Siebeck.

Popper, Karl R. (1959): The Logic of Scientific Discovery, New York: Basic Books.

Popper, Karl R. (1970): »Normal Science and Its Dangers«. In Alan Musgrave/Imre Lakatos (Hg.), Criticism and the Growth of Knowledge, Cambridge: Cambridge University Press, S. 51-58. 
Popper, Karl R. (1963): Conjectures and Refutations, London: Routledge and Kegan Paul.

Rheinberger, Hans-Jörg (1997): Toward a History of Epistemic Things. Synthesizing Proteins in the Test Tube, Stanford: Stanford University Press.

Sahlins, Marshall (1996): »The Sadness of Sweetness. The Native Anthropology of Western Cosmology«. Current Anthropology 37(3), S. 395428.

Strathern, Marilyn (2002): „Not Giving the Game Away«. In Andre Gingrich/Richard G. Fox (Hg.), Anthropology, by Comparison, London: Routledge, xiii-xvii.

Toulmin, Stephen (1970): »Does the Distinction between Normal and Revolutionary Science Hold Water?«. In Alan Musgrave/Imre Lakatos (Hg.), Criticism and the Growth of Knowledge, Cambridge: Cambridge University Press, S. 39-48

Virchow, Rudolf (1858): Die Cellularpathologie in ihrer Begründung auf physiologische und pathologische Gewebelehre. Zwanzig Vorlesungen, Berlin: August Hirschwald.

Watkins, J. W. N. (1970): »Against >Normal Science«». In Alan Musgrave/Imre Lakatos (Hg.), Criticism and the Growth of Knowledge, Cambridge: Cambridge University Press, S. 25-38.

Weisz, George M. (2010): »Dr Fleck Fighting Fleck Typhus«. Social Studies of Science 40(1), S. 145-153.

Young, Allan (1995): The Harmony of Illusions: Inventing Post-Traumatic Stress Disorder, Princeton: Princeton University Press. 